\title{
Queixas técnicas submetidas ao Sistema de Notificação e Investigação em Vigilância
}

\section{Sanitária}

\author{
Mariana Belincanta ${ }^{1}$, \\ Mariana Angela Rossaneis², \\ Laura Misue Matsuda ${ }^{3}$, \\ Alexsandro de Oliveira Dias ${ }^{4}$, \\ Maria do Carmo Lourenço Haddad ${ }^{5}$
}

\section{RESUMO}

Objetivou-se avaliar as queixas técnicas submetidas ao Sistema de Notificação e Investigação em Vigilância Sanitária entre 2006 a 2014. Pesquisa documental, transversal, quantitativa de um banco de dados secundário público. Identificou-se que das 245.940 notificações encaminhadas, 109.311 eram referentes a queixas técnicas. A região Sudeste apresentou maior repasse de informações $(53,5 \%)$ e a região Norte maior diferença na quantidade de notificações entre os Estados (coeficiente de variação= 159,2), seguida pela região Nordeste (coeficiente de variação= 124,8). A maioria dos casos de notificações foram de artigos médico-hospitalares $(53,09 \%)$ e o equipo foi o produto mais notificado (19\%). O risco à saúde do paciente foi identificado em 56.777 casos, com maior frequência quanto aos materiais de baixo ou médio risco. Os hospitais da Rede Sentinela foram os principais notificadores. No período analisado houve aumento anual gradativo no número de notificações de queixas técnicas, principalmente de artigos-médico-hospitalares utilizados em procedimentos invasivos.

Descritores: Vigilância de Produtos Comercializados; Notificação; Segurança de Equipamentos; Enfermagem; Segurança do Paciente.

\footnotetext{
${ }^{1}$ Enfermeira. Mestre em Enfermagem. Londrina, PR, Brasil. E-mail: mbelincanta@hotmail.com.

${ }^{2}$ Enfermeira, Doutora em Enfermagem. Professora Auxiliar da Universidade Estadual de Londrina. Londrina, PR, Brasil. E-mail: marianarossaneis@gmail.com.

${ }^{3}$ Enfermeira, Doutora em Enfermagem Fundamental. Professora Adjunta da Universidade Estadual de Maringá. Maringá, PR, Brasil. E-mail: lauramisuem@gmail.com.

${ }^{4}$ Enfermeiro. Doutor em Enfermagem Fundamental. Ribeirão Preto, SP, Brasil. E-mail: alexuel@bol.com.br.

${ }^{5}$ Enfermeira, Doutora em Enfermagem Fundamental. Professora Associada da Universidade Estadual de Londrina. Londrina, PR, Brasil. E-mail: carmohaddad@gmail.com.
}

\section{Como citar esse artigo:}

Belincanta M, Rossaneis MA, Matsuda LM, Dias AO, Haddad MCL. Queixas técnicas submetidas ao Sistema de Notificação e Investigação em Vigilância Sanitária. Rev. Eletr. Enf. [Internet]. 2018 [acesso em:

];20:v20a31. Disponível em: https://doi.org/10.5216/ree.v20.49337. 


\section{INTRODUÇÃO}

O desenvolvimento tecnológico e científico no âmbito da saúde e seus potenciais efeitos nocivos suscitaram a necessidade de monitorar a qualidade dos produtos disponíveis no mercado. Contudo nem todo o rigor empenhado no processo de pré-comercialização consegue prever as falhas dos produtos de interesse da saúde, uma vez que somente durante sua utilização, em circunstâncias reais e em grande escala, é que os eventos raros e os problemas não previstos podem ser identificados ${ }^{(1-2)}$.

Desse modo, a Vigilância Sanitária instituiu a Vigilância Sanitária Pós-Comercialização, ou Vigipós, com a premissa de desenvolver estratégias sensíveis para a detecção de queixas técnicas e eventos adversos durante as atividades laborais. Queixa técnica (QT) é caracterizada como o desvio de qualidade, presumido ou confirmado, detectado na empresa ou no produto, descrito por questões legais ou técnicas, com possibilidade de prejuízos individuais ou coletivos; enquanto evento adverso (EA) é o dano à saúde do indivíduo ou da coletividade ocasionado pelo uso habitual de um produto, desde que se respeitem as normas técnicas do fabricante ${ }^{(3-5)}$.

O dano evitável ao paciente é um grave problema de saúde pública e pesquisas apontam que este incidente já pode ter superado a doença cardíaca como a principal causa de morte nos Estados Unidos, considerando a subnotificação mesmo em países desenvolvidos ${ }^{(6)}$.

Estudos multicêntricos estimam que aproximadamente 10\% dos pacientes admitidos em unidades terciárias de saúde são vítimas de EA relacionados a materiais utilizados na área da saúde, e aproximadamente 60\% dos casos são decorrentes de fatos evitáveis. No Brasil, em 2011, de acordo com o Sistema de Informação Hospitalar do Sistema Único de Saúde, 7,6\% dos pacientes internados sofreram algum tipo de EA, com 66,7\% dos casos evitáveis e $40 \%$ de taxa de mortalidade. Números impactantes, a ponto de se inferir letalidade semelhante às decorrentes de AIDS, neoplasia mamária ou acidentes automobilísticos ${ }^{(7-8)}$.

No Brasil, em 2011, segundo o Sistema de Informação Hospitalar do Sistema Único de Saúde, 7,6\% dos pacientes internados sofreram algum tipo de EA, com $66,7 \%$ dos casos evitáveis e $40 \%$ de taxa de mortalidade. Esse impacto é significativo, a ponto de inferir mortalidade semelhante às decorrentes de AIDS, neoplasia mamária ou acidentes automobilísticos. Ainda, além da repercussão individual para a saúde do usuário, os EA provocam perdas financeiras consideráveis por aumentar o tempo de internação do indivíduo, por tratamentos adicionais aos danos decorrentes dos EA ou por indenizações judiciais ${ }^{(8-10)}$.

Diante desse panorama, a detecção e manejo adequado dos desvios de qualidade por meio das QT são fatores decisivos para a prevenção de EA e suas complicações. Uma das principais ferramentas utilizadas para este fim são as notificações de QT e EA submetidas ao Sistema de Notificação e Investigação em Vigilância Sanitária (Notivisa) $)^{(1)}$.

O Notivisa é uma plataforma on-line que sistematiza, investiga e gerencia os relatos de QT e EA fornecidos por serviços de saúde, profissionais liberais e instituições detentoras de registro de produtos. A referida plataforma recebe informações de materiais em vigilância sanitária nas seguintes categorias: tecnovigilância, farmacovigilância, hemovigilância e biovigilância ${ }^{(11)}$.

O uso desse sistema é fundamental para aumentar a eficiência e a eficácia dos serviços de saúde que visam a recuperação dos indivíduos com a minimização de danos e riscos ao seu bem estar. Nesse sentido o monitoramento, análise e interpretação das notificações de queixas técnicas, proporciona avanços na produção 
de materiais utilizados na assistência médico-hospitalar e abrange a área de pesquisa em sistemas de informação utilizados na gestão de saúde.

Em vista disso, este estudo propôs-se a responder a seguinte questão de pesquisa: quais características foram identificadas nas queixas técnicas submetidas ao Notivisa no período de 2006 a 2014? O objetivo deste estudo foi avaliar as queixas técnicas submetidas ao Notivisa no período de 2006 a 2014.

\section{MÉTODO}

Estudo documental, transversal, quantitativo, decorrente da análise de um banco de dados público das notificações de QT submetidas ao Notivisa no período de janeiro de 2006 a dezembro de 2014. 0 ano de 2006 foi selecionado considerando a implantação do Notivisa.

Foram analisadas as seguintes variáveis: ano da notificação, estado e região brasileira, categoria (medicamento, artigo médico-hospitalar, equipamento médico-hospitalar, cosmético, vacina e imunoglobulina, saneantes, kit reagente para diagnóstico in vitro e agrotóxico) e tipo do produto (equipo para infusão gravitacional de soluções parenterais, seringas descartáveis, luvas de procedimento e cirúrgicas etc), classificação (produto com suspeita de desvio de qualidade, produto com suspeita de estar sem registro, suspeita de empresa sem autorização de funcionamento, suspeita de outras práticas irregulares e suspeita de produto falsificado) e situação (concluída, em agrupamento, em análise, em investigação, enviada, análise pela empresa), categoria do notificador e classificação de risco referente às categorias de produto ou artigo médico-hospitalar. A coleta de dados ocorreu entre os meses de julho e outubro de 2015 e foi realizada pelo autor principal da pesquisa.

As variáveis "categoria do notificador" e "classificação de risco" foram reagrupadas. Para a primeira variável, as categorias foram: Rede Sentinela, profissional liberal, hospital com núcleo de segurança do paciente; os demais notificadores foram agrupados na categoria "outros". Para a segunda variável (classificação de risco), optou-se por trabalhar com três categorias: baixo/médio risco, risco misto e alto e máximo risco.

Os dados dos relatórios do Notivisa foram transcritos para planilhas do programa Microsoft Excel, em que, a partir das frequências absolutas, realizou-se análise descritiva com apresentação de medidas de tendência central (média) e de dispersão (desvio-padrão e coeficiente de variação).

Ainda para análise dos dados, solicitou-se ao Notivisa a relação do número de hospitais da Rede Sentinela em cada Estado brasileiro, referente a 2014. Além disso, fez-se busca no site do Instituto Brasileiro de Geografia e Estatística (IBGE) para coletar o total das populações de cada Estado naquele mesmo ano. Com base nessas informações, foram propostos dois indicadores, que, embora sejam indicadores iniciais, fornecem um panorama sobre a situação das notificações pautadas em algumas características específicas.

De acordo com o Notivisa, qualquer cidadão pode notificar uma QT, portanto, o primeiro indicador é um coeficiente de QT, considerando o total de QT dividido pelo total da população no mesmo período, multiplicado por $10^{n}$. Já o segundo indicador é um índice médio, considerando o total de QT pelo total de hospitais da Rede Sentinela nas regiões brasileiras.

Para análise da diferença entre as regiões brasileiras quanto às notificações, segundo a classificação de risco, calculou-se o teste de qui-quadrado, por meio do programa estatístico Bioestat 5.3. 
O projeto deste estudo foi aprovado pelo Comitê de Ética em Pesquisa da Universidade Estadual de Londrina-PR, sob o protocolo $\mathrm{n}$ ㅇ 1.356.669.

\section{RESULTADOS}

No período de 2006 a 2014, foram encaminhadas ao Notivisa 245.940 notificações. Destas, 109.311 (44,4\%) se referiam às QT. Nesse intervalo de tempo se observou crescimento gradativo desse tipo de notificações no território brasileiro, evoluindo de 88 casos em 2006 para 19.783 em 2014.

Entre as regiões brasileiras, a Sudeste foi a que mais contribuiu com o repasse das informações para a Vigilância Sanitária, com 53,5\% das notificações. Na região Norte, o maior número de notificações foi em 2009 , com 1.076 registros, decaindo nos demais anos; a região Centro-Oeste teve maior número de notificações em 2012 (923) (Figura 1).

Figura 1: Distribuição do número de notificações de queixa técnica por região, por ano, submetidas ao Sistema de Notificação e Investigação em Vigilância Sanitária (Notivisa).

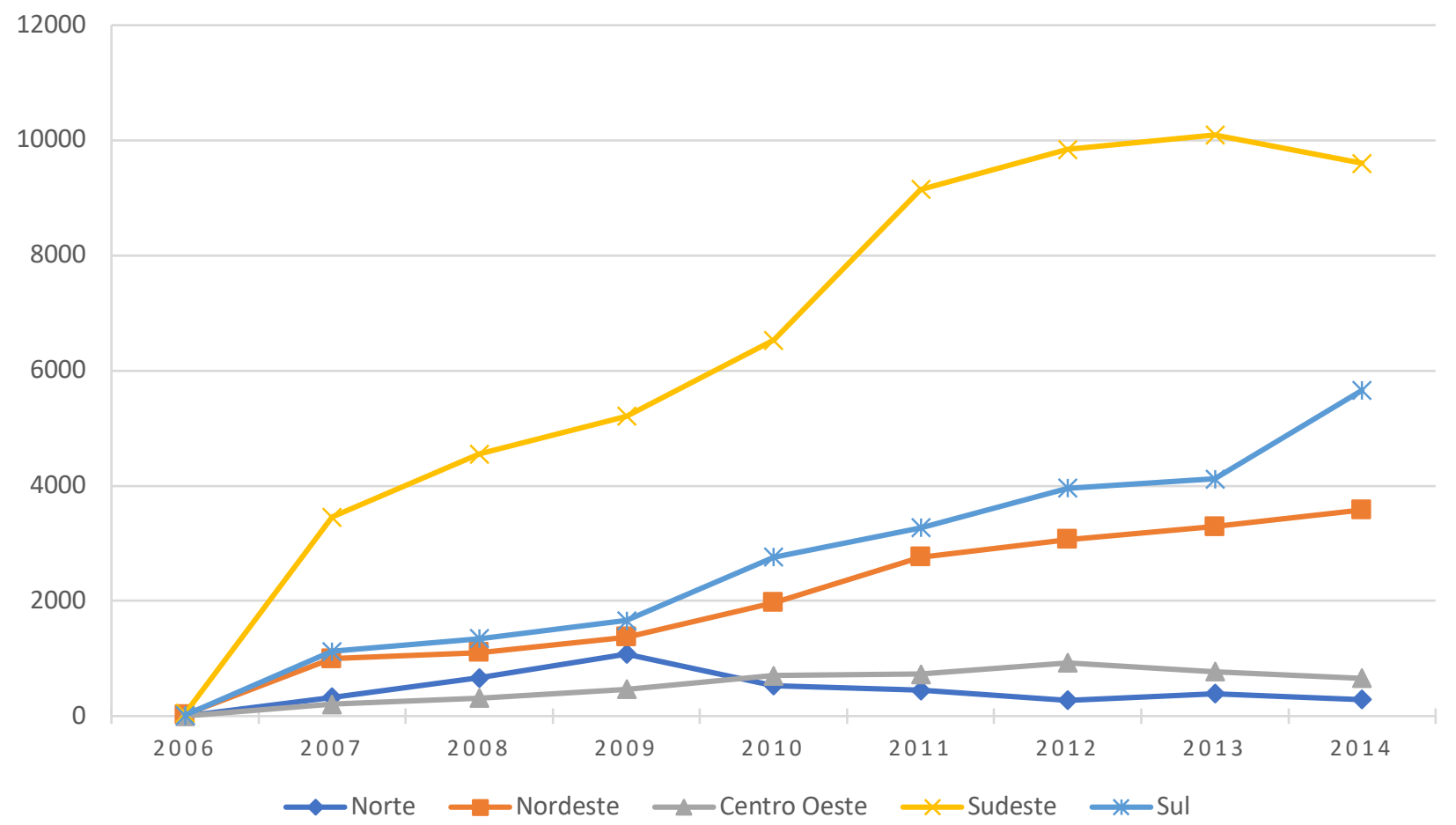

Na região Sudeste, os números mais elevados de notificações foram do Estado de São Paulo e Rio de Janeiro; na região Sul, os dois Estados com o maior número de notificações foram Santa Catarina e Paraná.

Por outro lado, Roraima e Amapá foram os Estados com o menor número de registro de QT. A região Norte apresentou maior instabilidade de notificações entre os Estados, seguida pela região Nordeste (Tabela 1).

Quanto aos produtos passíveis de notificação, constata-se que a parcela mais expressiva dessa categoria foram os artigos médico-hospitalares, com 53,09\% de casos, seguidos por medicamentos, com 40,30\%, e equipamentos médico-hospitalares, com 3,65\%, conforme demonstrado na Tabela 2. 
Tabela 1: Distribuição do número de notificações de queixa técnica por região e Estados brasileiros submetidas ao Sistema de Notificação e Investigação em Vigilância Sanitária. Brasil, 2006-2014.

\begin{tabular}{|c|c|c|c|c|c|}
\hline Região & Unidade federativa & N. de queixas técnicas & $\%$ & Média (dp) & Coeficiente de variação \\
\hline \multirow{5}{*}{ Sudeste } & São Paulo & 40.161 & 36,74 & \multirow{4}{*}{$14.616(17.523)$} & \multirow{5}{*}{119.9} \\
\hline & Rio Janeiro & 10.410 & 9,52 & & \\
\hline & Minas Gerais & 7.355 & 6,73 & & \\
\hline & Espírito Santo & 538 & 0,49 & & \\
\hline & Santa Catarina & 8.988 & 8,22 & \multirow{3}{*}{$7.969(1.292)$} & \\
\hline \multirow[t]{6}{*}{ Sul } & Paraná & 8.402 & 7,69 & & \multirow[t]{2}{*}{16.2} \\
\hline & Rio Grande do Sul & 6.516 & 5.96 & & \\
\hline & Ceará & 7.609 & 6.96 & \multirow{9}{*}{$2.019(2.521)$} & \multirow{9}{*}{124.8} \\
\hline & Bahia & 4.850 & 4,44 & & \\
\hline & Paraíba & 1.672 & 1,53 & & \\
\hline & Pernambuco & 979 & 0,90 & & \\
\hline \multirow[t]{5}{*}{ Nordeste } & Alagoas & 960 & 0,88 & & \\
\hline & Maranhão & 913 & 0,83 & & \\
\hline & Rio Grande do Norte & 654 & 0,60 & & \\
\hline & Piauí & 383 & 0,35 & & \\
\hline & Sergipe & 148 & 0,13 & & \\
\hline \multirow{4}{*}{ Centro-Oeste } & Distrito Federal & 3.167 & 2,90 & \multirow{4}{*}{$1.194(1.332)$} & \multirow{4}{*}{111.5} \\
\hline & Goiás & 741 & 0,68 & & \\
\hline & Mato Grosso do Sul & 613 & 0,56 & & \\
\hline & Mato Grosso & 254 & 0,23 & & \\
\hline \multirow{7}{*}{ Norte } & Pará & 2.589 & 2,37 & \multirow{7}{*}{571 (909) } & \multirow{7}{*}{159.2} \\
\hline & Amazonas & 518 & 0,47 & & \\
\hline & Tocantins & 430 & 0,39 & & \\
\hline & Acre & 246 & 0,22 & & \\
\hline & Rondônia & 158 & 0,14 & & \\
\hline & Amapá & 30 & 0,02 & & \\
\hline & Roraima & 27 & 0,02 & & \\
\hline
\end{tabular}

Fonte: Sistema de Notificação e Investigação em Vigilância Sanitária, 2006-2014.

Tabela 2: Distribuição do número de notificações de queixa técnica por produto/motivo submetidas ao Sistema de Notificação e Investigação em Vigilância Sanitária. Brasil, 2006-2014.

\begin{tabular}{ccc}
\hline Produto/Motivo & \multicolumn{2}{c}{ Queixa técnica } \\
\cline { 2 - 3 } Artigo médico-hospitalar & 58.032 & $\%$ \\
Medicamento & 44.054 & 53,09 \\
Equipamento médico-hospitalar & 3.993 & 40,30 \\
Cosmético & 1.287 & 3,65 \\
Saneantes & 998 & 1,18 \\
Kit reagente para diagnóstico in vitro & 817 & 0,91 \\
Vacina e imunoglobulina & 129 & 0,75 \\
Agrotóxico & 01 & 0,12 \\
\hline Total & $\mathbf{1 0 9 . 3 1 1}$ & 0,00 \\
\hline
\end{tabular}

Fonte: Sistema de Notificação e Investigação em Vigilância Sanitária, 2006-2014

Dentre os artigos médico-hospitalares, os sete produtos com as maiores frequências de notificações de QT no Notivisa foram: equipos (17,95\% de), seringas descartáveis (10,45\%), luvas de procedimento e cirúrgicas $(9,68 \%)$, cateteres $(6,17 \%)$, compressas $(3,79 \%)$, sondas $(3,59 \%)$ e agulhas descartáveis $(2,71 \%)$.

Em relação à classificação das notificações enviadas ao Notivisa, a maioria classificou-se como suspeita de desvio de qualidade (95,18\%), seguida por suspeita de outras práticas irregulares (2,87\% dos casos). Quanto à 
situação dessas notificações no Sistema, 41,43\% foram concluídas e 58,57\% estavam em processo de análise pela Anvisa.

$\mathrm{Na}$ análise dos notificadores, verificou-se que os três maiores responsáveis pelo registro e envio de QT foram: hospitais da Rede Sentinela, profissionais liberais de hospitais que possuem Núcleos de Segurança do $(12,3 \%)$. Convém destacar que essa distribuição é diferenciada, de acordo com as regiões brasileiras, pois se verifica que nas regiões Sudeste, Sul, Norte e Nordeste os hospitais da Rede Sentinela são os responsáveis pela maior frequência de notificações (Tabela 3).

Tabela 3: Distribuição do número de notificações de queixa técnica por região e por categoria do notificador, submetidas ao sistema Sistema de Notificação e Investigação em Vigilância Sanitária. Brasil, 2006-2014.

\begin{tabular}{cccccc}
\hline \multirow{2}{*}{ Notificadores } & Sudeste & Sul & Centro-Oeste & Nordeste & Norte \\
\cline { 2 - 6 } & $\mathbf{N ~ ( \% )}$ & $\mathbf{N ~ ( \% )}$ & $\mathbf{N}(\%)$ & $\mathbf{N}(\%)$ & $\mathbf{N}(\%)$ \\
\hline Rede Sentinela & $35.521(60,7)$ & $11.197(46,8)$ & $1.101(23,0)$ & $10.256(56,45)$ & $3.155(78,9)$ \\
Profissional liberal & $6.951(11,8)$ & $2.835(11,8)$ & $1.042(21,8)$ & $2.929(16,1)$ & $452(11,3)$ \\
Hospital com NSP** & $6.188(10,5)$ & $3.56(14,8)$ & $467(9,7)$ & $313(17,2)$ & $144(3,6)$ \\
Outros notificadores & $9.804(16,7)$ & $6.314(26,4)$ & $2.165(45,3)$ & $1.853(10,2)$ & $247(6,1)$ \\
\hline Total Geral & $\mathbf{5 8 . 4 6 4 ( 5 5 , 4 )}$ & $\mathbf{2 3 . 9 0 6 ( 2 1 , 8 )}$ & $\mathbf{4 . 7 7 5 ( 4 , 3 )}$ & $\mathbf{1 8 . 1 6 8 ( 1 6 , 6 )}$ & $\mathbf{3 . 9 9 8 ( 3 , 6 )}$ \\
\hline
\end{tabular}

Fonte: Sistema de Notificação e Investigação em Vigilância Sanitária, 2006-2014.

Embora a região Sudeste apresente as maiores frequências de notificação de QT, o maior número de hospitais da Rede Sentinela o coeficiente de QT mais elevado foi verificado na região Sul. Por outro lado, na região Norte, mesmo com um número reduzido de hospitais da Rede Sentinela, a média de notificações de QT foi a terceira maior entre as regiões brasileiras, abaixo apenas das médias das regiões Sudeste e Sul (Tabela 4).

Tabela 4: Distribuição do coeficiente de queixa técnica por habitantes e do índice de notificação pelos hospitais da Rede Sentinela de acordo com as regiões que submeteram notificações ao Sistema de Notificação e Investigação em Vigilância Sanitária. Brasil, 2006-2014.

\begin{tabular}{ccccccc}
\hline Região & QT & Habitantes & Coeficiente QT & QTE/RS & HRS & Média QT/HRS \\
\hline Sudeste & 58.464 & 85.339 .316 & 0,68 & 35.521 & 103 & 344,9 \\
Sul & 23.906 & 29.074 .721 & 0,82 & 11.197 & 43 & 260,3 \\
Nordeste & 18.168 & 56.200 .315 & 0,32 & 10.256 & 46 & 222,9 \\
Centro-Oeste & 4.775 & 15.285 .867 & 0,31 & 1.101 & 11 & 100,1 \\
Norte & 3.998 & 17.305 .188 & 0,23 & 3.155 & 12 & 262,9 \\
\hline Total & $\mathbf{1 0 9 . 3 1 1}$ & $\mathbf{2 0 3 . 2 0 5 . 4 0 7}$ & $\mathbf{0 , 5 4}$ & $\mathbf{6 1 . 2 3 0}$ & $\mathbf{2 1 5}$ & $\mathbf{2 8 4 , 8}$ \\
\hline
\end{tabular}

Legenda: QT: Queixa técnica, QTE: Queixa técnica específica; RS: Rede Sentinela; HRS: Hospitais da Rede Sentinela.

Fonte: Sistema de Notificação e Investigação em Vigilância Sanitária, 2006-2014.

Considerando a classificação de risco referente às categorias de produto e artigo médico-hospitalar (Tabela 5), foram relatados 56.777 casos, com maior frequência dos materiais de baixo ou médio risco $83,44 \%$ das notificações). Procedeu-se a comparação entre as distribuições das notificações de queixas técnicas nas regiões, de acordo com a classe de risco dos produtos, por meio do teste qui-quadrado e verificou-se uma diferença estatisticamente significativa $(p<0,001)$.

As regiões Sudeste, Sul e Nordeste obtiveram distribuições semelhantes quando comparados os riscos, variando de $82,62 \%$ a $84,68 \%$ das notificações na categoria de baixo/médio risco, e de $14,59 \%$ a $15,70 \%$ para alto/máximo risco. Embora com frequência absoluta menor em relação às regiões Sul, Sudeste e Nordeste, a 
região Centro-Oeste foi a que proporcionalmente gerou o maior número de notificações na categoria de produtos considerados como de alto/máximo risco (Tabela 5).

Tabela 5: Distribuição das notificações de queixa técnica de equipamento e artigo médico-hospitalar de acordo com a região, por classe de risco de produtos para a saúde submetidos ao Sistema de Notificação e Investigação em Vigilância

Sanitária. Brasil, 2006-2014.

\begin{tabular}{|c|c|c|c|c|c|c|c|}
\hline \multirow{2}{*}{ Região } & \multicolumn{2}{|c|}{ Baixo / Médio Risco } & \multicolumn{2}{|c|}{ Risco Misto } & \multicolumn{2}{|c|}{ Alto / Máximo Risco } & \multirow[b]{2}{*}{ p-valor } \\
\hline & $\mathbf{N}$ & $\%$ & $\mathbf{N}$ & $\%$ & $\mathbf{N}$ & $\%$ & \\
\hline Sudeste & 25.040 & 82,62 & 507 & 1,67 & 476 & 15,70 & \\
\hline Sul & 9.610 & 84,49 & 75 & 0,65 & 1.688 & 14,84 & \\
\hline Nordeste & 9.012 & 84,68 & 78 & 0,73 & 1.553 & 14,59 & 0,000 \\
\hline Norte & 2.235 & 90,41 & 8 & 0,32 & 229 & 9,26 & \\
\hline Centro-Oeste & 1.478 & 74,57 & 41 & 2,07 & 463 & 23,36 & \\
\hline
\end{tabular}

Fonte: Sistema de Notificação e Investigação em Vigilância Sanitária, 2006-2014.

\section{DISCUSSÃO}

A evolução tecnológica e o constante desenvolvimento de produtos utilizados para assistência ao paciente exige medidas efetivas de vigilância em saúde para garantir a segurança sanitária daqueles que vão manusear e/ou consumir esses produtos, pois, muito além dos benefícios, o uso excessivo de tecnologias traz em si um potencial iatrogênico, com mediação de novos riscos individuais e coletivos que desafiam os sistemas de regulamentação e controle sanitário mundial(12).

As atividades destinadas a monitorização de queixas técnicas e eventos adversos não se restringem ao Brasil. Inúmeros países também estão voltados a atividades de notificação como estratégia para analisar os desvios de qualidade e minimizar danos como, por exemplo, os Estados Unidos da América (EUA) e Membros da União Europeia que já vislumbravam este cenário há muitos anos(13).

Os EUA adotaram um dos mais rigorosos sistemas de controle sanitário, sendo responsabilidade do Food and Drug Administration (FDA), vinculado ao US Department of Health and Human Services, o monitoramento de medicamentos (humano e animal), cosméticos, equipamentos médicos, materiais biológicos, produtos derivados do sangue humano, alimentos (tanto humano como animal) e suplementos alimentares. O FDA foi fundado em 1862, com a atual designação a partir de 1930, com o objetivo de controlar a qualidade dos itens supralistados e garantir a segurança individual e coletiva frente à comercialização destes materiais ${ }^{(14)}$.

Neste país, verifica-se dois processos norteadores, que englobam os requisitos de ensaios e pesquisas para registro na fase pré-comercialização (Premarket) e o acompanhamento dos produtos disponíveis no mercado em uma fase de pós-comercialização (Postmarket). São implementadas estratégias para avaliar cientificamente as relações dos eventos adversos e os riscos potenciais com o uso de determinado produto, fato que permite a retroalimentação das equipes de saúde durante a análise, revisão e revalidação dos registros ${ }^{(14)}$.

Já na União Europeia (UE), o órgão responsável por executar as atividades de vigilância à saúde é o CHAFEA (Costumers, Health, Agriculture and Food Executive Agency) em parceria com a EMA (European Medicine Agency). Estas agências executam suas atividades da perspectiva de promover a saúde e a segurança dos de pacientes e coletividade, minimizando a ocorrência de eventos adversos, por meio da avaliação e investigação dos incidentes 
relatados e divulgando relatórios e alertas quando necessário. Esse sistema de vigilância facilita a correlação das informações entre as autoridades nacionais competentes e as empresas responsáveis por determinado material, propiciando ações corretivas precoces ${ }^{(14)}$.

$\mathrm{Na}$ análise dos dados, foi possível identificar aumento progressivo do número de notificações de QT desde a implantação do Notivisa em 2006, fato que reforça a legitimidade dessa estratégia sanitária. Estudos realizados no período de 2006 a 2013 confirmam o crescente relato de QT: em 2006, foram reportados 10.543 ocorrências, atingindo 15.228 casos em 2008 e tornando-se mais evidente em $2013^{(10-11,15)}$. Em contrapartida, outros estudos demonstram oscilações entre os anos de 2007 e 2009, com queda de até 14,5\% nas notificações registradas em $2009^{(16-17)}$.

Embora os dados analisados demonstrem aumento gradual no número de relatos, deve-se considerar a possibilidade de subnotificações, de forma subjetiva, uma vez que ocorreu significativa variação no número de notificações no decorrer do período. Há também a possibilidade de que algumas ocorrências tenham sido investigadas e concluídas por órgãos de vigilância sanitária estadual ou municipal e não tenham sido incluídas no sistema Notivisa ${ }^{(18)}$.

De fato, mesmo as notificações de investigação obrigatórias referentes a EA, tais como as de óbito, lesão permanente ou temporária grave, somente serão investigadas caso seja possível identificar o produto suspeito e o detentor do seu registro. Além disso, é preciso levantar evidências para caracterizar a causalidade, o que reforça a necessidade de que as notificações sejam completas e coerentes. Um estudo demonstrou que, em $53 \%$ dos eventos reportados à unidade hospitalar, não constavam informações quanto aos sinais e sintomas apresentados pelo paciente e, em $49 \%$ dos casos, não foram descritas as lesões causadas pelo $\mathrm{EA}^{(10)}$. Outro estudo constatou que, em 2009, cerca de $61 \%$ das notificações não continham a data da ocorrência, dificultando o acompanhamento destes casos $^{(16)}$.

Em contrapartida, é fato que em muitas instituições a cultura de segurança tem sido promovida e promovem o desenvolvimento de estratégias para aquisição de produtos de maior qualidade para assistência. Assim como, capacita os profissionais para estarem em constante fiscalização para identificação de materiais com potencial para oferecer risco aos usuários e trabalhadores ${ }^{(19)}$.

Quanto à região brasileira e suas unidades federativas, a região Sudeste precedida das regiões Sul e Nordeste foram as que mais contribuíram para o repasse de informações, porém uma pesquisa ${ }^{(20)}$ revelou que, em 2007, o município de Belém, localizado na região Norte, realizou 7\% e 22,1\% das notificações de QT e EA, respectivamente, colocando-se como a quinta capital brasileira a reportar QT e a segunda quanto a EA.

Considerando o coeficiente de variação a respeito da distribuição demográfica, observa-se que a região Sul apresenta maior homogeneidade de notificações entre seus Estados, com o menor coeficiente de variação entre as regiões brasileiras. Embora a região Sudeste seja a maior notificadora de QT, verifica-se significativa heterogeneidade entre seus Estados, com alusão à distribuição irregular da Rede Sentinela, sendo 61 unidades no Estado de São Paulo e apenas uma unidade no Estado de Minas Gerais. Por outro lado, a região Norte, que possui o maior coeficiente de variação, apresenta elevada discrepância entre seus Estados, principalmente entre o Pará e Roraima, que efetuaram 2.589 e 159 notificações, respectivamente, no período do estudo. Essa divergência 
pode mais uma vez refletir altas taxas de subnotificação, visto que, em ambos os Estados, há apenas quatro unidades da Rede Sentinela(18).

Entre 2006 e 2011, foram submetidos ao Notivisa 118.106 relatos de QT e EA, sendo 37.696 relativos a medicamentos; 29.880 a artigos médico-hospitalares, 19.105 a sangue e seus componentes e; 27.406 a intoxicações $^{(9-10)}$. Analisando isoladamente as QT notificadas nesse mesmo período, observa-se que os artigos médico-hospitalares lideraram as ocorrências, seguidos dos medicamentos ${ }^{(2,4)}$.

No presente estudo, identificou-se que os equipos de infusão foram responsáveis pelo maior número de notificações de QT. Tal resultado se assemelha a outros estudos ${ }^{(10,17)}$, em que esse artigo foi o que mais recebeu notificação por QT e EA. O dano na estrutura do equipo ou na sua embalagem podem causar danos graves ao paciente visto que é utilizado para procedimentos invasivos. Desta forma supõe-se ainda que os profissionais que utilizam este material identificam as falhas técnicas como mais graves e por isso tendem a notificar com maior frequência. Ademais, destaca-se que o equipo constitui um dos artigos médico-hospitalares utilizados expressivamente na rotina hospitalar e, mesmo que tenha valor comercial unitário relativamente baixo, o amplo consumo gera impactos financeiros significativos quando analisados em grande escala ${ }^{(11,21)}$.

No que se refere ao tipo de QT, verificou-se que a maioria dos casos dizia respeito a produto com suspeita de desvio de qualidade, tendo-se constatado dado similar em outro estudo, em que $97,10 \%$ das notificações de QT estavam ligadas à suspeita de desvio de qualidade e apenas 0,5\% estava relacionada ao uso inadequado do produto $^{(12)}$.

Pela análise de estudos ${ }^{(11,16)}$, grande parcela das empresas detentoras de registo $(10,10 \%)$ apresenta produtos destinados à saúde com qualidade insatisfatória, uma vez que $60,80 \%$ desses materiais apresentaram múltiplas notificações ${ }^{(16)}$.

Vale ressaltar que o emprego de produtos com desvio de qualidade reflete também no ônus hospitalar, visto que os custos diretos e indiretos com os insumos à saúde impactam em $35 \%$ a $45 \%$ do orçamento geral da instituição ${ }^{(3)}$. Desse modo, é de responsabilidade da empresa detentora do registro garantir a qualidade do produto, corrigir e prevenir a recorrência das falhas identificadas e/ou propor alterações do projeto, a fim de garantir a segurança do usuário de acordo com os requisitos das "Boas Práticas de Fabricação de Produtos Médicos"(22).

A maioria dos relatos que foram submetidos ao Notivisa ainda está em processamento e, de acordo com a gravidade do caso, foi desencadeado o processo investigativo. Notificações que não são imediatamente investigadas são mantidas em um banco de dados até que uma análise de tendência demonstre sua importância ou, ainda, um aumento no número de notificações suficiente para desencadear a abertura de inquérito(16-17).

As instituições de saúde exercem papel crucial para a consolidação da vigilância sanitária intra-hospitalar e a Rede Sentinela é a protagonista no repasse de informações ao Notivisa ${ }^{(2,12)}$. Em 2010, essa rede estava distribuída no território brasileiro com aproximadamente 26 unidades na região Norte, 55 na região Nordeste, 16 na região Centro-Oeste, 107 na região Sudeste e 43 na região Sul. Era responsável por 59,8\% das notificações, seguidas por $14,3 \%$ dos profissionais liberais e $8,3 \%$ dos hospitais ${ }^{(10)}$.

Os hospitais da Rede Sentinela exercem papel fundamental no monitoramento dos produtos disponíveis no mercado, visto que são qualificados para detectar QT e EA. Há, porém, regiões onde o processo de implantação 
dessas unidades necessita de aprimoramento, como a região Centro-Oeste, que ainda conta expressivamente com outras fontes para o repasse de informação. A região Norte, embora não represente o notificador mais expressivo, utiliza como principal observatório a Rede Sentinela, fato que comprova sua efetividade mesmo em ambientes menos favorecidos. Essa rede contribui para a divulgação do panorama nacional e, em alguns momentos, mundial dos insumos comercializados, permitindo a cada hospital colaborador apropriar-se de ações corretivas e preventivas que fomentem a minimização de riscos individuais e coletivos ${ }^{(16)}$.

Refletindo ainda sobre a efetividade da implantação da Rede Sentinela em algumas regiões, observa-se que a região Sudeste permaneceu na liderança quanto ao índice médio de QT por hospital da Rede Sentinela, com 103 unidades distribuídas na maior população brasileira. Entretanto, a região Norte, que possui apenas 12 unidades, apresentou a segunda maior média de notificações, seguida pela região Sul, com 43 unidades, fato que demonstra a importância e o comprometimento da rede nos mais diversos ambientes.

Considerando a classificação de risco no tocante às categorias de produto e artigo médico-hospitalar, no presente estudo se evidenciou que a maioria dos produtos notificados pertencia à classe de risco baixo ou médio. Esses resultados se assemelham aos encontrados na literatura, em que os produtos de médio risco foram responsáveis por grande parte das notificações de QT, seguidos pela classe de risco máximo ${ }^{(22)}$.

Em consonância, um estudo ${ }^{(16)}$ identificou que $72 \%$ dos produtos notificados apresentavam risco médio à saúde e à segurança do paciente. Dentre esses, 33,80\% foram representados por equipos, 7,20\% por seringas, $4,80 \%$ por luvas, $4,80 \%$ por sondas e 3,30\% por agulhas. Constatou-se também que $4,80 \%$ dos produtos foram classificados como risco máximo à saúde e à segurança do paciente e daqueles que os manuseiam.

Vale ressaltar que estudos dessa magnitude são pioneiros, pois publicações nesta área ainda são escassas, fato que instiga o aprimoramento de novas pesquisas e indicadores mais sensíveis para análise do real impacto das notificações de QT em nível nacional, bem como a investigação das subnotificações - que são mais ostensivas onde a implantação da Rede Sentinela não é efetiva - além da criação de indicadores que retratem o panorama real do comprometimento de todos os profissionais envolvidos no processo de monitoramento da qualidade dos produtos empregados na saúde ${ }^{(2,11)}$.

\section{CONCLUSÃO}

Este estudo demonstrou que houve aumento gradativo no número de notificações no território brasileiro, consolidando o sistema como forma de monitoramento de produtos sujeitos à vigilância sanitária. Observou-se que a Vigipós demonstrou perspicácia ao planejar uma estratégia como a Rede Sentinela, mesmo que algumas regiões ainda se encontrem muito incipientes em todo esse processo, ressaltando a importância da análise crítica do contexto sanitário nacional.

Entre os produtos passíveis de notificações, os produtos médico-hospitalares se destacaram pelo maior número de notificações, sendo o equipo o artigo com a maior frequência de QT. Quanto à situação dessas notificações no Notivisa, a maioria estava sendo investigada e analisada pela Anvisa.

As notificações de queixas técnicas ao Notivisa mostraram-se relevantes para gerar um indicador de qualidade, e até mesmo garantir melhores produtos de saúde no mercado e mais segurança para pacientes e profissionais de saúde, além de orientar tomadas de decisões. Nesse sentido, faz-se necessário implementar 
estratégias que estimulem a adesão dos profissionais envolvidos na prestação do cuidado para realizarem as notificações de QT e, assim, contribuírem para a redução da ocorrência de subnotificações.

Vale salientar que a importância do processo de notificação reside no fato de possibilitar o reconhecimento de não conformidades, que tanto podem gerar QT como EA, e que a prevenção da ocorrência desses acontecimentos deve ser considerada prioridade de todas as pessoas envolvidas nesse processo, desde 0 desenvolvimento do produto até a sua utilização.

Deste modo, por meio deste estudo espera-se contribuir para o despertar das boas práticas de segurança nos profissionais de saúde e sugere-se a realização de estudos que investiguem outros fatores envolvidos no processo de notificação de QT, tais como número de instituições de saúde, leitos hospitalares e profissionais da área, a fim de auxiliar na elaboração de indicadores mais sensíveis para notificação das QT no cenário brasileiro.

A limitação deste estudo consiste nas dificuldades relacionadas as pesquisas com fontes de dados secundários, como a subnotificação das queixas técnicas. No NOTIVISA as notificações são espontâneas e dependem da ação das instituições. Por isso é necessário as constantes campanhas de conscientização dos gestores e profissionais de saúde para a notificação de queixas técnicas e eventos adversos.

\section{REFERÊNCIAS}

1. Acauan LV, Rodrigues MCS, Pereira JL. Gestão da qualidade em centro de diagnóstico por imagem e segurança do paciente: um ensaio reflexivo. Ciênc. cuid. Saúde [Internet]. 2014 [acesso em: 06 dez. 2018];13(1):179-84. Disponível em: http://eduem.uem.br/ojs/index.php/CiencCuidSaude/article/view/19239.

2. Manfredi MAB, Menoncin SM, Santos TG. Tecnovigilância: a qualidade dos produtos para saúde em hospital da rede sentinela. Latin American Journal of Pharmacy [Internet]. 2010 [acesso em: 06 dez. 2018];29(4):626-30. Disponível em:

http://www.latamjpharm.org/resumenes/29/4/LAJOP $2942 \quad 6$.pdf.

3. Gil RB, Chaves LDP, Laus AM. Gerenciamento de recursos materiais com enfoque na queixa técnica. Rev. Eletr. Enf. [Internet]. 2015 [acesso em: 06 dez. 2018];17(1):100-7. Disponível em: https://doi.org/10.5216/ree.v17i1.27544.

4. Azulino ACO, Costa MHA, Carvalho MN, Moreira AS, Oliveira AF, Pinto AG, et al. Queixas técnicas realizadas pelos profissionais da saúde, relacionadas aos produtos utilizados em Hospital Sentinela de Belém - Pará. Revista Brasileira de Farmácia Hospitalar e Serviços de Saúde [Internet]. 2013 [acesso em: 06 dez. 2018];4(3):13-6. Disponível em:

http://www.sbrafh.org.br/rbfhss/index/edicoes/vl/4/nr/3/id/442/lg/0.

5. Lima PF, Cavassini ACM, Silva FAT, Kron MR, Gonçalves SF, Spadotto A, et al. Queixas técnicas e eventos adversos a medicamentos notificados em um hospital sentinela do interior de São Paulo, 2009-2010. Epidemiol Serv Saude [Internet]. 2013 [acesso em: 06 dez. 2018];22(4):679-86. Disponível em: https://doi.org/10.5123/S1679-49742013000400014.

6. Card AJ. Patient safety: this is public health. J Healthc Risk Manag [Internet]. 2014 [acesso em: 06 dez. 2018];34(1):6-12.

Disponível em: https://doi.org/10.1002/jhrm.21145.

7. Silva LD. Safety and quality in Brazilian hospitals. Rev. enferm. UERJ [Internet]. 2013 [acesso em: 06 dez. 2018];21(4):425-6.

Disponível em: https://www.e-publicacoes.uerj.br/index.php/enfermagemuerj/article/view/10037/15732.

8. Reis CT, Martins M, Laguardia J. A segurança do paciente como dimensão da qualidade do cuidado de saúde: um olhar sobre a literatura. Cien Saude Colet [Internet]. 2013 [acesso em: 06 dez. 2018];18(7):2029-36. Disponível em:

https://doi.org/10.1590/S1413-81232013000700018.

9. Silva LA, Terra FS, Macedo FRM, Santos SVM, Maia LG, Batista MHJ. Notificação de eventos adversos: caracterização de eventos ocorridos em uma instituição hospitalar. Revista de Enfermagem UFPE on line [Internet]. 2014 [acesso em: 06 dez. 2018];8(9):301523. Disponível em: https://periodicos.ufpe.br/revistas/revistaenfermagem/article/view/10020.

10. Oliveira JR, Xavier RMF, Santos Júnior AF. Eventos adversos notificados ao Sistema Nacional de Notificações para a Vigilância Sanitária (NOTIVISA): Brasil, estudo descritivo no período 2006 a 2011. Epidemiol Serv Saude [Internet]. 2013 [acesso em: 06 dez. 2018];22(4):671-8. Disponível em: https://doi.org/10.5123/S1679-49742013000400013.

11. Reis GAX, Rossaneis MA, Haddad MCFL, Gil RB, Pissinati PDSC. Análise das notificações de queixa técnica de material médicohospitalar em um hospital sentinela. Vigilância Sanitária em Debate: Sociedade, Ciência \& Tecnologia [Internet]. 2016 [acesso em: 06 dez. 2018];4(1):52-57. Disponível em: https://doi.org/10.3395/2317-269x.00588.

12. Turbelin C, Boëlle PY. Exposing public health surveillance data using existing standards. Stud Health Technol Inform [Internet]. 2013 [acesso em: 06 dez. 2018];192:802-6. Disponível em: https://doi.org/10.3233/978-1-61499-289-9-802. 
13. Groseclose SL, Buckeridge DL. Public Health Surveillance Systems: Recent Advances in Their Use and Evaluation. Annu Rev Public Health [Internet]. 2017 [acesso em: 06 dez. 2018];38:57-79. Disponível em: https://doi.org/10.1146/annurev-publhealth031816-044348.

14. Sutherland JM, Busse R. Canada: Focus on a country's health system with provincial diversity. Health Policy [Internet]. 2016 [acesso em: 06 dez. 2018];120(7):729-31. Disponível em: https://doi.org/10.1016/j.healthpol.2016.06.010.

15. Schmitt MD, Haddad MCFL, Rossaneis MA, Pissinati PSC, Vannuchi MTO. Análise das notificações de queixas técnicas em tecnovigilância em hospital universitário público. Vigilância Sanitária em Debate: Sociedade, Ciência \& Tecnologia [Internet]. 2016 [acesso em: 06 dez. 2018];4(3):35-41. Disponível em: https://doi.org/10.22239/2317-269x.00750.

16. Morais LO, Friedrick K, Melchior SC, Silva MF, Gemal AL, Delgado IF. Eventos adversos e queixas técnicas relacionados ao fio para sutura cirúrgica comercializado no Brasil. Vigilância Sanitária em Debate: Sociedade, Ciência \& Tecnologia [Internet]. 2013 [acesso em: 06 dez. 2018];1(2):35-43. Disponível em: https://doi.org/10.3395/vd.v1i2.25.

17. Varallo FR, Guimarães SOP, Abjaude SAR, Mastroianni PC. Causes for the underreporting of adverse drug events by health professionals: a systematic review. Rev Esc Enferm USP [Internet]. 2014 [acesso em: 06 dez. 2018];48(4):739-47. Disponível em: https://doi.org/10.1590/S0080-623420140000400023.

18. Silva JPB, Santos DC, Meireles HAS, Souza FM, Aguiar ASS, Melgaço TB, Maia CSF. Análise do sistema de notificações em vigilância sanitária - NOTIVISA. Revista Paraense de Medicina [Internet]. 2012 [acesso em: 06 dez. 2018];26(4). Disponível em: http://www6.ensp.fiocruz.br/visa/?q=node/5933.

19. Weaver SJ, Lubomksi LH, Wilson RF, Pfoh ER, Martinez KA, Dy SM. Promoting a culture of safety as a patient safety strategy: a systematic review. Ann Intern Med [Internet]. 2013 [acesso em: 06 dez. 2018];158(5 Pt 2):369-74. Disponível em: https://doi.org/10.7326/0003-4819-158-5-201303051-00002.

20. Kuwabara CCT, Évora YDM, Oliveira MMB. Risk Management in Technovigilance: Construction and Validation of a MedicalHospital Product Evaluation Instrument. Rev Lat Am Enfermagem [Internet]. 2010 [acesso em: 06 dez. 2018];18(5):943-51.

Disponível em: https://doi.org/10.1590/S0104-11692010000500015.

21. Trindade E, Vicente MG, Hofmeister MGSA, Cruz CJF, Hayashi ELO, Mathos MC, et al. Estudo multicêntrico de pré-qualificação: estudo de caso sobre equipos. Boletim informativo de tecnovigilância [Internet]. 2010 [acesso em: 06 dez. 2018];(esp.). Disponível em: http://portal.anvisa.gov.br/resultado-de-

busca? $p \quad p \quad i d=101 \& p \quad p$ lifecycle $=0 \& p \quad p$ state $=$ maximized\& $p \quad p$ mode $=v i e w \& p \quad p \quad$ col $i d=$ column-

$1 \& p \_p \_c o l \_c o u n t=1 \& \_101$ struts action=\%2Fasset_publisher\%2Fview_content\&_101_assetEntryld=327533\&_101_type=content \& 101 groupld=33868\& 101 urlTitle=boletim-informativo-de-tecnovigilancia-bit\&inheritRedirect=true.

22. Branco NMC, Lopes RGA, Silva MF, Romão CMAP. NOTIVISA e os Laboratórios de Saúde Pública: A interface da informação em Vigilância Sanitária. Vigilância Sanitária em Debate: Sociedade, Ciência \& Tecnologia [Internet]. 2015 [acesso em: 06 dez.

2018];3(3):130-4. Disponível em: https://doi.org/10.3395/2317-269x.00242. 